\title{
Beyond the axioms: The question of objectivity in mathematics
}

\author{
W. W. Tait*
}

I will be discussing the axiomatic conception of mathematics, the modern version of which is clearly due to Hilbert; ${ }^{1}$ but one aspect of it goes back to Cantor. I refer here to Hilbert's view that the demands of mathematical existence and truth are entirely met by a suitable axiomatization. By whatever dialectical process we come to adopt the axioms, whatever 'intuitions' lead us to them, the question of mathematical truth or existence becomes welldefined only with the introduction of the axioms. Now Cantor is one of the last people, ever, to cite in support of the axiomatic method; but Hilbert's point about mathematical existence and truth is a natural consequence of the discussion in Foundations of a General Theory of Manifolds [Cantor, 1883] of free mathematics and in particular of the limited obligation of free mathematics to establish only the immanent existence of its objects. Speaking of this in connection with his transfinite numbers, he wrote

*I read versions of this paper at The George Boolos Memorial Symposium in Logic and the Foundations of Mathematics at Notre Dame, April 15-18, 1998 and in a workshop in Tübingen in January, 1999; and the present version has profited very much from the subsequent discussions, in particular from remarks by Richard Heck and Mark Wilson at Notre Dame. Although the paper was written in memory and honor of George Boolos, I am not at all sure that he would have approved of the topic. Certainly his own work displays a taste for those parts of philosophy that can be carved out and made precise. I sensed in him, especially in our relatively small correspondance, an impatience with those topics in philosophy, like the one I am addressing, which seem to carry the possibility of infinite ambiguity.

${ }^{1}$ In fact, it seems clear that this conception goes back to the time of Plato. See my [1992], [1986a] and [n.d.] and §4, below. 
First, we may regard the whole numbers as real in so far as, on the basis of definitions, they occupy an entirely determinate place in our understanding, are well distinguished from all other parts of our thought and stand to them in determinate relationships, and thus modify the substance of our minds in a determinate way.

Leaving aside the heavily psychologistic formulation, the important message in this quote is that all that is demanded is a coherent account of the relationships among the numbers. ${ }^{2}$ We have here the modern origin, as far as I know, of the view that the question of objectivity in mathematics concerns, not primarily the existence of objects, but the objectivity of mathematical discourse. Objectivity in mathematics is established when meaning has been specified for mathematical propositions, including existential propositions $\exists x F(x)$. This obviously resonates with Frege's so-called context principle, ${ }^{3}$ although Frege seems to have rejected the general view point of Cantor and, more explicitly, Hilbert towards mathematical existence. On the other hand, his conflicts with Hilbert in this respect seem to relate to his unique view of geometry and are beside my present point. In any case, Hilbert certainly goes beyond both Cantor and Frege in specifying how the meaning of the propositions is to be fixed, namely by means of an axiom system. With the axioms in place, what objects exist and what is true of them become questions of what can be logically deduced from the axioms.

When I speak of the axiomatic method or of axioms here, I don't mean axioms of abstract mathematics such as group theory or geometry, but rather those axioms which we posit for 'concrete' mathematics - axioms of logic and the theory of finite and transfinite numbers and the cumulative hierarchy of sets over them - in other words, that part of mathematics in which we can reason about arbitrary groups, spaces and the like, and can construct examples of them. For, obviously, the question of objective existence and truth concern only this part. But it is precisely concerning this part of mathe-

\footnotetext{
${ }^{2}$ Cantor's reference to 'other parts of our thought' and their relationship with the transfinite numbers seems to concern the finite whole numbers. It is required that we be able to distinguish them from the transfinite numbers and that we be able to extend the arithmetic of finite numbers to the transfinite, while distinguishing those laws which hold for the one and not for the other.

${ }^{3}$ However, Frege's point is deeper: Cantor and Hilbert were concerned only with mathematical existence. Frege based his view on a general - though certainly abbreviatedanalysis of meaning, which applies equally outside the domain of mathematics. This part of Frege's thought is developed convincingly in Wittgenstein's Philosophical Investigations.
} 
matics that the axiomatic method seems to run into difficulties. For one thing, if meaning and truth are to be determined by what is deducible from the axioms, then we ought to require at least that the axioms be consistent, since otherwise the distinctions true/false and existence/non-existence collapse. But we know that there is no relevant sense in which we can be sure that this requirement is met. No matter how familiar we become with set theory, or even arithmetic, as at least partially expressed by axioms, and no matter how intuitive those axioms are or become for us, consistency is just something that, ultimately, we must take on faith. And if we should discover a contradiction in Peano Arithmetic, say, that would not show that numbers do not exist: rather it would undermine the sense of existence assertions concerning numbers (and so the sense of their negations, as well). So I will simply leave the question of consistency aside, noting only that if inconsistency existed at a sufficiently low level, it would be a problem also for natural science, i.e., for our conception of existence in the natural world.

Another requirement for the axioms, if they are to determine truth, might seem to be that they be complete; and, indeed, consistency and completeness were precisely the conditions that Hilbert placed on axiom systems. If these two conditions could be met, it would provide a very satisfactory conception of mathematics: no matter what intuitions we may have about the natural numbers or the reals, say, with a consistent and complete axiom system, we can have no fear that they will not be captured in the axioms; for if our intuitions are consistent and tell us that the axioms are true, then all of the intuitive truths will follow from the axioms. But, of course, completeness fails and must fail. Nor is the essential incompleteness due simply to Gödel's incompleteness theorem. Since Cantor's introduction of the transfinite numbers in [1883] with the principle that every set of numbers has a least upper bound, mathematics and, in particular, the notion of a set of transfinite numbers has been open-ended. So, even leaving aside the matter of consistency, the simple and rather attractive picture that Hilbert's axiomatic conception provided for mathematics is compromised. Beyond the axioms that we have accepted as properly analyzing our notions of number, set, function, etc., there remain, and always will, propositions which are undecided by them.

Independent of these technical difficulties with the axiomatic conception, 
there has been another, 'philosophical', objection to it, which rejects not only Hilbert's axiomatics, but also Cantor's underlying conception of free mathematics and immanent existence. ${ }^{4}$ This objection is that, even though the axioms might be entirely consistent and in their consequences express, as far as they go, our conception of numbers, sets, etc., they could nevertheless be false or meaningless because the objective universe of numbers and sets is just not like that or, worse, does not even exist. The view that there is an external criterion of mathematical existence and truth and that numbers, functions, sets, etc., satisfy it, is often called 'Platonism'; but Plato deserves a better fate. Wittgenstein, at least in analogous cases, called it 'realism'; but I want to save this term for the view that we can truthfully assert the existence of numbers and the like without explaining the assertion away as saying something else. Realism in this sense is the default position: When one believes mathematics is meaningful and has, as one inevitably must, finally become convinced that mathematical propositions cannot be reduced to propositions about something else or about nothing at all, then one is a realist. For example, Cantor, Dedekind, Frege and Hilbert were realists in this sense, without advocating the more extreme position. In [*1951], Gödel argues explicitly for realism in this default sense; but it is likely that, at least on some occasions, he went beyond this and held the more extreme position. Let me refer to the extreme position as super-realism. An important consequence of super-realism and, as I believe, a telling objection to it, is that it implies an alienation of truth in mathematics from what we actually do: mathematics become speculative in the sense that even the most elementary computations, deductions and propositions must answer to a reality which we, at best, can only partially comprehend and about which we could be wrong. If there are grounds for truth and existence and they are not the axioms, then the axioms could be false.

Aside perhaps from Gödel, I don't think that super-realism is a view explicitly held by any of the renowned figures in the late-nineteenth and twentieth century discussion of foundations of mathematics. More often, it is identified with realism by opponents of the latter on the fallacious grounds

\footnotetext{
${ }^{4}$ I am using the term "philosophical" here not in a sense that I approve, but to refer to alleged issues about the sciences which nevertheless stand at a different level from them. From this stance, for example, there can be doubts about the legitimacy of the science which are in no way manifested in the science itself. Roughly, my use of the term coincides with Wittgenstein's when, in the Philosophical Investigations, he is referring to what other philophers do.
} 
that realism implicitly implies super-realism. For example, both Cantor and Frege have been called Platonists on these grounds, dispite their explicit explanations of their positions. In at least one case, it has been argued that super-realism is a consequence of the use of non-constructive reasoning in mathematics, so that its only alternative is constructive mathematics. I have argued against this in [1983] and, more generally, against the identification of super-realism with realism elsewhere (see $[1983 ; 1986 \mathrm{~b}]$ ); but perhaps, in view of a wide-spread tendency to conflate them under the common heading 'Platonism', it is worth briefly considering a typical description of the latter. I quote from Ruben Hersh's characterization of Platonism in What is Mathematics, Really? [Hersh, 1997, p.11, p.138], with interpolated comments.

Mathematical objects are real.

In one sense, this may be simply an expression of realism: this is the sense in which we judge, for example, that there is a prime number strictly between $n$ and $2 n$ for arbitrary positive integers $\mathrm{n}$ without reconstruing it to be saying something which makes no reference to mathematical objects. In another sense, it may be an expression of super-realism: in this sense, we imagine that it makes sense to question the validity of mathematics - say, of arithmetic - over all, not because of some internal defect in it, but because as a matter of fact - not a mathematical fact, as we ordinarily understand it, but a super-mathematical fact - there really are no numbers at all. Following Wittgenstein, I have argued that this external denial of existence makes no prime faci sense [1986a]. Of course it follows that the external affirmation of existence is equally without content.

Their existence is an objective fact, independent of our knowledge of them.

From the realist perspective, what is objective are the grounds for judging truth of mathematical propositions, including existential ones, namely, derivability from the axioms. But to hold that there is some ground beyond this, to which the axioms themselves are accountable, is to enter the domain of super-realism, where mathematics is again speculative. For, again, if the axioms are accountable, then they might be false.

Infinite sets, uncountably infinite sets, infinite dimensional manifolds, space-filling curves - all the denizens of the mathematical zoo-are definite objects, with definite properties. Some of their properties are known, some are unknown. 
Interestingly, the examples are all infinite objects, although I should think that even finite objects, such as the null set, would be a challenge to one who wants to question whether mathematical objects are 'real'. Of course one may contemplate the possibility of reconstruing mathematical propositions about finite things, say propositions of elementary number theory, as really not being about mathematical objects at all. But Gödel's challenge [*1951] remains unanswered to actually give a satisfactory such reconstrual. ${ }^{5}$ Secondly, the notion of 'definiteness' applied to objects is not really very clear. How can an object not be definite? Of course a description of an object might not be definite; but we can give a description of the infinite set of finite ordinals and of the uncountably infinite set of countable ordinals which are definite in the only sense that I can reasonably assign to that notion: there is a proof that one and only one set satisfies the description. If one rejects the proof, that is not a case of rejecting the definiteness of an object, but rather of calling into question whether any object at all has been picked out by the description. The same holds true, for example, for a space-filling curve defined by some equation, relative to a specification of a coordinate system for the space.

It is possible that, in speaking of definiteness, Hersh has in mind that the language and axioms of arithmetic, for example, although they determine the system of numbers to within isomorphism, do not pick out one particular simply infinite system. So, when we speak of ' 2 ', we might mean $\{\{\emptyset\}\}$, if we are thinking of Zermelo's original representation of the finite ordinals, or $\{\emptyset,\{\emptyset\}\}$, if we are thinking of von Neumann's. This is the problem that Benacerraf discussed in [1974]. But, as Dedekind argued in his well-known letter to Weber of 1888, one should not confuse a representation of the numbers with the numbers themselves [Dedekind, 1932, p.490]. 2 is a number, not a set; and so it is neither $\{\{\emptyset\}\}$ nor $\{\emptyset,\{\emptyset\}\}$.

The reference to definite properties is not entirely clear. If it means that for every property $P(x)$, say, of numbers and every number $n$, either $P(n)$ is true or it is false, then Hersh later on repeats it more explicitly:

A meaningful statement about one of these objects is true or false, whether we know it or not.

Let me leave this aside for the moment: I will argue below that this 'definiteness' amounts only to using the laws of classical logic, in particular the law of

\footnotetext{
${ }^{5}$ In this connection, one might consult [Burgess and Rosen, 1997], for an extended discussion and assessment of nominalist strategies in mathematics.
} 
excluded middle, in reasoning about numbers. In this sense, the assumption of definiteness is compatible with realism. On the other hand, "with definite properties" could possibly refer to the assumption that the totality of truths about numbers is 'definite' in the sense of being a well-defined extension. This assumption goes beyond realism, since the latter is compatible with the view that what is true in mathematics is what can be proved; and, given the essential incompleteness of mathematics, this is an open-ended notion, depending on what new axioms we admit.

That Hersh should include the assertion that "some of their properties are known, some are unknown" as an ingredient of Platonism is puzzling: that there is an infinitude of primes is known and it is not known whether there is an infinitude of prime pairs. Any conception of mathematical knowledge which fails to admit this is clearly deficient.

These objects aren't physical or material. They're outside space and time. They're immutable. They're uncreated. Mathematicians are empirical scientists, like botanists. We can't invent anything; its already there. We try to discover.

All of this is not only compatible with realism in the default sense but is of its essence, understood properly as a grammatical statement. To say that mathematical objects are "outside of space and time" means that spatial and temporal properties simply aren't meaningfully predicable of them; and all the rest follows: that they are not physical or material is a consequence of their not being in space or time, and that they are immuitable and uncreated follows from the fact that mutation and creation take place in time. Of course, one may feel that the properties of being changeable or created only apply to things in time, in which case we would want to say that it makes no sense even to speak of the mutability or creation of mathematical objects. On the other hand, it is sometimes reasonable to speak of their being discovered in the sense that, for example, Cantor discovered the transfinite numbers sometime in 1882. But there seems to me to be some problem with speaking of the discovery of the natural numbers or Euclidean space: when were they discovered, and by whom?

Our mathematical knowledge is objective and unchanging because it's knowledge of objects external to us, independent of us, which are indeed changeless. 
Speaking from a point of view which is realistic in the default sense, our mathematical knowledge, in the sense of what is known, is objective, in that the criterion for truth, namely provability, is public: it is there for us all to apply. Of course, the criterion depends on the fact that we agree about what counts as a correct application - what counts as a correct proof; but, nevertheless, there is such agreement and the criterion is the same for all and is in no way subjective. But surely what is known is subject to change: either collectively or individually, we continue to learn new mathematics. Moreover, the admission of new axioms leads to the possibility of new knowledge which was not possible on the basis of previously admitted axioms.

As for the objects themselves, numbers and the like, they are, as we have already noted, indeed changeless in the sense that temporal properties simply don't apply to them. Are they independent of us? If humans did not exist, number theory would not exist either, being our creation. But it would be a category mistake to infer from this that the existence of numbers depends on our existence. The proposition $P \longrightarrow \exists x(x$ is a prime number) is true for any proposition $P$, including the proposition that no human beings exists. Finally, what in the world has Hersh in mind when he speaks of them being 'external' to us? Presumably, he means that they are not in our minds; but what would be the alternative to numbers, for example, not being in our minds? In whose mind would they be? All of ours? All of the time?

I hope that it is clear that much of what Hersh takes to be characteristic of Platonism, when it is not simply silly, can be understood to express nothing more than grammatical facts about the language of mathematics, so long as we take this language literally and do not attempt to translate it into another language, about other things. Having criticized Frege in [1997a] for attacking too much peoples' choice of words rather than the substance of what they say, I feel somewhat sensitive about this point in connection wilth my treatment of Hersh (and others) here; but there is a difference. Many of Frege's victims were saying something sensible, which Frege either misunderstood or misrepresented. Those who speak of Platonism in the same vein as Hersh undoubtedly feel that they are saying something different from or at least more than the rather deflationary assertions that I am drawing from Hersh's words. But that is precisely what I am challenging, that there is another genuine sense to be given his words. There is indeed a doctrine that goes beyond realism in the sense I am speaking of: what I am calling 
super-realism. But Hersh's discussion is far too careless to separate the two. ${ }^{6}$

\section{III}

Let me return now to the problem that incompleteness poses for Hilbert's realism. One problem that has been raised is that, if $A$ is an undecidable proposition, then nevertheless, $A \vee \neg A$ is provable and so, by our criterion, true; but it is alleged that, by our criterion, neither $A$ nor $\neg A$ is true. This consideration has been behind the assumption that realism, in so far as it embraces classical (non-constructive) logic, must collapse to super-realism. What is at issue is a distinction between an instance $A \vee \neg A$ of the law of excluded middle and an instance, ( $A$ is true) $\vee(\neg A$ is true), of the so-called principle of bivalence; and the argument is that on the provability criterion for truth, when $A$ is undecidable, then the instance of excluded middle is true in classical logic, but the instance of bivalence fails, contrary to the classical meaning of disjunction.

Note that this argument presupposes that there is a difference in meaning between " $B$ " and " $B$ is true" for a mathematical proposition $B$, and so a real difference between excluded middle and bivalence. But we are not speaking of truth here in the sense of a sentence of some formal language being true

\footnotetext{
${ }^{6}$ In this respect, Hersh does not stand alone, nor is the carelesness the province only of amateur philosophers. For example, in a paper entitled "Platonism", Dummett writes [1978, 202]

Platonism, as a philosophy of mathematics, is founded on a simile: the comparison between the apprehension of mathematical truth [and] the perception of physical objects, and thus of mathematical reality to the physical universe. For the platonist, mathematical statements are true or false independently of our knowledge of their truth-values: they are rendered true or false by how things are in the mathematical realm.
}

And from Burge's paper "Frege on Knowing the Third Realm" we have

Platonism, as I understand the doctrine, regards some entities ... as existing non-spatially and atemporally. Further it avoids commenting on them as having special status, including being dependent for their existence or nature (as opposed to their discovery) on practise or mental activity. They are in no way derivative, instrumental, fictional or otherwise second class. [Burge, 1992, p.6] 
in a model: we are speaking of the truth of a mathematical proposition. For example, let $B$ be an elementary arithmetical proposition and let $\phi$ be its formalization in the language of first-order arithmetic. We may define in the usual way the truth predicate for sentences in this formal language in the intended model $\mathcal{N}=\langle N, 0, S,+, \times\rangle$. But when we now unpack the proposition " $\phi$ is true in the model $\mathcal{N}$ " according to the definition of truth, we see that it essentially expresses the same thing as $B .{ }^{7}$ How can " $B$ is true" express something different? The fact is that truth, in the sense of truth of a mathematical proposition (as opposed to truth of a formal sentence in a structure), plays no role in mathematics. But if we are to give it meaning, how could we reasonably assign a meaning to the proposition that $B$ is true that is more or less than the proposition $B$ itself? ${ }^{8}$ It follows then that there is no difference between excluded middle and bivalence: " $A$ is true or $A$ is false" expresses precisely that " $A$ or $\neg A$ ". 9

One source of confusion about this arises, for example, from the assertion above that provability is the criterion for truth. If one takes this to mean that " $B$ is true" is defined to mean " $B$ is provable" from some particular axioms, then the existence of undecidable sentences does yield a distinction between excluded middle and bivalence. Of course, when I say that provability is the criterion for truth, I don't mean provability from some particular axioms. Rather I am saying that, in mathematics, the assertion of truth is justified only by a proof, whatever we take the basis of the proof, the axioms, to be. Nevertheless, even if we take truth to mean what in the long run we would count as provable and assuming that there will be substantial

\footnotetext{
${ }^{7}$ Of course, if the truth predicate is defined by way of satisfaction, then " $\phi$ is true" will be of higher order than $B$. But if we take advantage of the fact that every number $n$ is named by the numeral $\bar{n}$, so that e.g. " $\forall x \psi(x)$ is true" is defined to mean $\forall x(\psi(\bar{x})$ is true), then " $\phi$ is true" and $B$ are literally the same proposition, assuming we use the same mathematical symbols in the metalanguage as in the object language.

${ }^{8}$ Perhaps behind the view that there is a difference lies a picture of the domain of all mathematics, existing as a kind of supermodel, and that the truth of an ordinary mathematical proposition $B$ is expressed in the language of this super-model, i.e. in the super-metalanguage. But this would again be an expression of super-realism: The criterion for truth - and even meaningfulness - would no longer be in ordinary mathematical practice, but would stand above it.

${ }^{9}$ Hence my remark above in response to Hersh's statement that Platonism involves the assumption that every "meaningful statement about one of these objects is true or false, whether we know it or not"; namely that this assumption involves nothing more than the employment of classical logic in mathematics.
} 
agreement about this, excluded middle and bivalence would still be distinct properties and the assertion that they are extensionally equivalent would be an unwarranted expression of optimism. However, my assertion that provability is a criterion for truth is not intended as a definition of truth. Rather, it is a convenient and natural way to express the fact that the assertion of a mathematical proposition is warranted only by a proof of it. Of course, this notion of warrant is open-ended; but that is simply an expression of the essential incompleteness of mathematics; it is not a further problem arising from it.

A more serious challenge that incompleteness poses to objectivity on the axiomatic conception of mathematics concerns how we are to understand undecidable propositions with respect to future determinations. Take for example the continuum hypothesis $\mathrm{CH}$ and suppose that the future development of mathematics in the form of new axioms leads to a particular decision about this, say that $\mathrm{CH}$ is true. Does it make sense to ask whether these new axioms are correct or incorrect? Again, let's leave aside the question of consistency: if our present axioms of set theory are consistent, then there are consistent extensions leading to $\mathrm{CH}$ and to $\neg \mathrm{CH}$. The problem is that, on the axiomatic conception, the axioms define the subject matter and so there is no criterion for truth beyond what can be proved from the axioms. So, on this view, there is no issue of truth concerning a proposed new axiom prior to its acceptence. Now, especially in view of the essential open-endedness of mathematics, this may seem a bitter pill to swallow; but I think that there are considerations in favor of it, as well as considerations concerning its alternative, that make it more palatable. The first thing to note is that this position does not amount to saying that $\mathrm{CH}$ is neither true nor false. We have already disarmed this objection by noting that the assertion that $\mathrm{CH}$ is either true or false amounts to nothing more than the application of the law of excluded middle to it.

But, moreover, to say that there is no external source of correctness for proposed new axioms is not to say that there are no grounds for accepting them. It can happen that, examining the consequences of a proposed new axiom, we see that it yields a theory more natural or fruitful or more in keeping 
with our intuitions than its alternative - in the way that we probably want to say that Hilbert's axiomatization of Euclidean geometry is a more reasonable development of Euclid's Elements than its alternatives. Similarly, maybe we will arrive at an understanding of the notion of set according to which it will seem reasonable to adopt an axiom which decides $C H$. Again, successively stronger axioms of infinity may suggest themselves to us as naturally following from our conception of the sequence of transfinite numbers. It could even be that there is something in us, some common bit of hardwiring, so to speak, which, together with environmental factors, determines that we will adopt this or that axiom. But, even if this were so, it yields only an empirical explanation of our acceptance of the axiom, not a justification of the axiom, itself.

Allow me to mention that it is precisely the process of examination, leading to the adoption of new 'first principles', mentioned above, that Plato called "dialectic" and which he distinguished from purely deductive reasoning, which proceeds from the first principles. Indeed, Hilbert's axiomatic conception of mathematics is the natural development of Plato's conception of exact science as noesis, as a priori, which is the core of real 'Platonism' (as opposed to the way in which the term is currently used). Incidently, it goes without saying that adopting the axiomatic conception of mathematics is merely adopting a criterion for mathematical truth and existence: it is emphatically not a criterion for doing mathematics. Clearly the process of finding the right definitions and axioms, the work of Plato's dialectician, is equally important as deriving the consequences of these principles.

It may seem that Plato was a super-realist, after all, since the Forms seem to stand for him as the criteria of truth of the axioms. But it is not at all clear that he has to be read in that way. What is clear is that he appealed to the Forms to account for the fact that we can have an understanding of structures, e.g. geometric objects such as points, curves and surfaces, finite sets ('arithmoi'), etc., which, although they are imperfectly exemplified in experience, are not really given in experience. What we understand, what leads us to the choice of first principles, is what he calls the Forms. It is the autonomy of reason, our ability to reason about these 'separate' Forms independently of experience, that impressed him. It is, perhaps, a moot point whether or not he thought that the Forms were exhausted by the first principles, in the sense that these principles simply express what we had in mind, as opposed to the view that we have some other kind of knowledge of the Forms against which our first principles may be measured - and could 
possibly be found to be in error. But I think this was his view: after all, he wrote of seeking the truth of the things that are (i.e., empirical things) in logoi, not in the Forms ${ }^{10}$.

But, as with Hilbert (initially), this question may not have been important for Plato, since he could contemplate the possibility that we would find all the first principles and so, agreeing on them, there would be nothing more to discuss. The issue of the possible 'justification' of first principles, axioms, has become more meaningful for us, today, in the face of incompleteness where, by the possibility of justification, remember, I am not referring to the intuitions or dialectical considerations that may lead us to accept a new axiom, but rather the possibility of some ground upon which independent propositions are true independently of these intuitions or dialectical grounds. The position that, in general, there are such grounds would seem to be just another affirmation of super-realism. For the same grounds, whatever they are, upon which a proposition, undecided by our present axioms, is nevertheless really true or really false would seem to be grounds upon which the axioms themselves are really true or really false. If mathematics is not to be speculative, then it seems that we have to give up the view that there is some 'correct way' that it can develop. Here, I should say, I am agreeing with one possible sense of Feferman's assertion [1997] that " $\mathrm{CH}$ is indeterminate", although I don't agree with his accompanying minimalist conception of mathematics. Until we determine it, $\mathrm{CH}$ is indeed indeterminate; but that doesn't mean that there can be no natural grounds, if only we seek them, on which to determine it. Of course, it needn't necessarily be the case that there is just one direction in which our conception of set can develop; and so to say that there may be natural grounds on which to decide $\mathrm{CH}$ one way does not mean that there may not also be natural grounds upon which to decide it the other way.

In any case, incompleteness, even the essential incompleteness of mathematics, is not grounds for abandoning Hilbert's realism. As long as there remains an objective criterion for truth, namely provability from the axioms,

\footnotetext{
${ }^{10}$ Phaedo, 99e5-6
} 
it is inessential to this conception that there always remain some indeterminate propositions. However, there is a further challenge to realism which seems to cut deeper, because it challenges the idea that provability from the axioms is objective. I refer here to what Jon Barwise has called the "black plague of post-modernism" ${ }^{11}$ Crudely stated, it is this: There is a clear sense in which mathematics, like language, is a cultural construct. The norms of correctness are grounded in the practice of the community. But then how can there be mathematical truths beyond what the society has explicitly stipulated? And how can there be absolute mathematical truths in any sense, i.e. truths that are not relative to the culture that stipulates them? These questions of course generalize from the case of mathematical and logical norms to other norms, such as moral and aesthetical norms. For it seems equally clear that all such norms are social constructs. So the question in general is how there can be truths whose basis seems to be social constructions and yet which society has not explicitly stipilated. And, how can there be absolute truths of any kind on such bases? Why is not a normative statement a statement about what someone or everyone or, at least, everyone of a certain class does or assents to?

I am going to discuss only the case of mathematics, though I think that what I have to say has implications also for other 'cultural relativities'. It should be noted that 'cultural relativity' in this sense in the mathematical case concerns not just the question of what axioms are to be accepted, but also - and from our present perspective, more importantly - of what we are to do with them. Of course, we have rules of inference which tell us which inferences may be drawn from the axioms; but if we now ask for rules to tell us how to apply the rules of inference, we are drawn into an infinite regress. At the end of the day, we simply have to say that there is agreement on how we do it, on how we follow the rules, make inferences, etc. This would seem to undermine the objectivity that we claim for the notion of proof from axioms, for it argues that what counts as a valid inference is to be understood entirely in terms of our cultural practice. It would certainly seem to undermine the necessity that we ascribe to mathematical truth, since it is a contingent fact that our practice is this and not that.

On the other hand, the reaction that meaning and truth are indeed objective and that our practice simply conforms to the objectively given again leads to super-realism and the question: Are we sure that we are conforming?

\footnotetext{
${ }^{11}$ A posting 21 Dec 1997 13:16:12 to the email list FOM on foundations of mathematics.
} 
Thus, again, mathematics becomes speculative.

It may seem that the two horns of the dilemma in this case are not exhaustive. A third possibility may suggest itself, namely that, although mathematical concepts come to us clothed in our mother tongue and we come to understand them by being trained in how to do things - compute, prove, etc.- - this cultural acquisition is merely an external trapping, essential for coming to possess, perhaps, but not of the essence of possessing mathematical concepts. Once I have grasped the concept, it is my grasp, what $I$ mean, that determines what is true of it and what is false. Communication between you and me, on this conception, is always a case of a handshake across our individual ideolects. This ideocentric view underlies Brouwer's philosophy of mathematics and there are perhaps traces of it in Gödel's writings. It would seem to be the basis of any conception according to which individual intuitions are grounds for mathematical truth. But, however attractive this view may initially appear to be, it doesn't really survive being spelled out; and I believe that it is utterly undermined by Wittgenstein's critique of rulefollowing in, for example, his Philosophical Investigations.

The problem is in trying to identify a notion of private grasp of the concept which will bear the weight of being the source of meaning and truth concerning it. Let me mention the prima faci reasonable candidates for such a notion and very briefly indicate why they seem to be inadequate to the task.

Understanding consists of a definition or a mental picture.

Each of these needs, itself, to be understood; and so we are in an infinite regress. In the case of a definition, my grasp of the concept depends on my prior grasp of the concepts occuring in the definition; and in the case of a mental picture, it itself stands in need of interpretation, in the sense of an explanation of how it is to be applied.

Understanding consists in some mental state.

Well, we might discover that a certain scale of brain states correlates well with performance; and so we might begin to consider brain scans as a substitute for addition quizes and, maybe, if the coincidence became firmly established the term "understanding addition" would come to refer to a certain range 
in the scale of brain states; much as color words came to refer to the corresponding wave lengths. But in the case of understanding, whatever we may reasonably expect to eventually discover, at the moment there is no sufficient knowledge of an underlying cause, physiological or mental, of one's ability to add numbers. For us, the term "understanding" refers primarily to a propensity or more specifically to a competence - for example to our ability to add numbers correctly. However, even if we were to discover a causal mechanism underlying the competence to add, say, it would still be the case that neither the competence itself nor the causal mechanism can account for the difference between correct and incorrect addition. The laws of evolution of a machine are not teleological: one can't ascribe purpose or norms to the machine. It is true that the terms "machine" and "mechanism" seem to imply a purpose; but that purpose is supplied from without; it is a question of what we want the machine to do. I am disposed to add as I do, whether or not there is a causal explanation for it; but neither my disposition nor its underlying cause accounts for why what I do counts as correct or incorrect. The very term "competence" entails the existence of an external source of norms, against which to judge particular instances of adding as correct or incorrect. In short, understanding is not itself the source of meaning or truth; rather the question of whether or not one understands itself presupposes meaning: we understand if we act in accordance with the meaning - e.g. we add numbers correctly.

Incidently, I think that this argument bears on Fodor's idea of a private language, a language of thought as he calls it. On his view, we are all supposed to embody such a language, say it is a mental implimentation of some Turing machine. But, again, the implementation, the mental machine, contains in itself no criteria for correct use of language. There is of course a natural source of norms for this mental machine, namely the Turing machine, i.e. the mathematical object, which it implements. But this is no longer a private language, its the language which, on Fodor's hypothesis, we all speak.

So, getting back to the point, it seems that the two horns of the dilemma stand: meaning is either culturally determined, which seems to make meaning and truth relative to the culture, or else there is a standard of meaning and truth independent of us and our culture, leaving open the possibility that ordinary mathematical practice, computing, proving and the like, may be invalid or even meaningless. We have rejected the later horn and so are stuck with the former.

But nevertheless, we are not therefore stuck with the 'black plague of 
postmodernism', with the loss of objective truth in mathematics and, in particular, the loss of objective validity in making inferences. Ironically, it is Wittgenstein, who most convincingly argued for the dilemma, who also pointed the way out of this presumed consequence of it. Perhaps the most forthright expression of his argument is in Philosophical Investigations §241:

"So you are saying that human agreement decides what is true and what is false?" - It is what human beings say that is true or false; and they agree in the language they use. That is not agreement in opinions but in a form of life.

So, in judging an inference valid or invalid, we are not making an assertion about what we in general do, what we have done in the past or will do in the future. Rather it is that making inferences, judging them valid and the like are all activities that are founded on our agreement in, let us say, a form of life. If there were no such agreement, it wouldn't follow that what we now call valid inferences would be invalid; rather, there would be no institution of mathematics and, in particular, there would be no notion of logical validity.

However, one might feel that this argument does not entirely answer the postmodern attack on objectivity. For, as we stated initially, what is objective should be independent of what we, singly or collectively, believe. To quote Frege [1893]

[B] eing true is different from being taken to be true, whether by one or many or everybody, and in no case is to be reduced to it. There is no contradiction in something's being true which everybody takes to be false.

But it might seem that, according to the argument just given, we are still in the position of having to say that, if we didn't in general agree that an inference is valid, then it would not be valid. But I think that this is wrong and is based on a confusion that has prevailed both in the secondary literature on Wittgenstein and in postmodern thought about the consequences of the banal fact that norms, logical or otherwise, are cultural constructions. There is a sense in which it is not the case that, if we didn't in general agree that a certain (in fact) valid inference is valid, then it would not be valid. That is precisely the same counterfactual sense in which we might not have the form of life, the cultural practice, that we in fact have. For the reference point of notions of validity and logical and mathematical necessity is the 
practice that we in fact have. In the sense in which I am speaking of it, that practice cannot change, although we may change our practice. Thus Frege is absolutely right: There is no contradiction in an inference being valid that everybody takes to be invalid. For there is no contradiction in the supposition that we have a different form of life from the one we in fact have.

\section{References}

Benacerraf, P. (1974). What numbers could not be, Philosophical Review 74: 47-73. Reprinted in [Benacerraf and Putnam, 1983, 272-294].

Benacerraf, P. and Putnam, H. (eds) (1983). Philosophy of Mathematics: Selected Readings, second edn, Cambridge University Press. First edition 1964.

Burge, T. (1992). Frege on knowing the third realm, Mind 101: 634-650. Reprinted in [Tait, 1997].

Burgess, J. and Rosen, G. (1997). A Subject with no Object: Strategies for Nominalistic Interpretation of Mathematics, Oxford: Oxford University Press.

Cantor, G. (1883). Über unendliche, lineare Punktmannigfaltigkeiten, 5, Mathematische Annalen 21: 545-586.

Dedekind, R. (1932). Gesammelte Werke, vol. 3, Braunschweig: Vieweg. Edited by R. Fricke, E. Noether, and O. Ore.

Detlefsen, M. (ed.) (1992). Proof and Knowledge in Mathematics, London: Routledge.

Dummett, M. (1978). Truth and Other Enigmas, Cambridge: Harvard University Press.

Feferman, S. (1997). Does mathematics need new axioms?, American Mathematical Monthly 106: 99-111.

Frege, G. (1893). Grundgesetze der Arithmetik: Begriffsschriftlich abgeleitet, Band I, Jena: H. Pohle. Reprinted in 1962 along with [Frege, 1903], Hildesheim:Georg Olms. 
Frege, G. (1903). Grundgesetze der Arithmetik: Begriffsschriftlich abgeleitet, Band II, Jena: H. Pohle.

Gödel, K. (*1951). Some basic theorems on the foundations of mathematics and their implications, [Gödel, 1995].

Gödel, K. (1995). Collected Works, Vol. III, Oxford: Oxford University Press.

Hersh, R. (1997). What is Mathematics, Really?, Oxford: Oxford University Press.

Tait, W. (1983). Against intuitionism:constructive mathematics is part of classical mathematics, Journal of Philosophical Logic 12: 175-195.

Tait, W. (1986a). Plato's second best method, Review of Metaphysics 39: $455-482$.

Tait, W. (1986b). Truth and proof: the Platonism of mathematics, Synthese 69: $341-370$.

Tait, W. (1992). Reflections on the concept of a priori truth and its corruption by Kant, In [Detlefsen, 1992] pp. 33-64.

Tait, W. (1997a). Frege versus Cantor and Dedekind: on the concept of number, In [Tait, 1997] pp. 213-248.

Tait, W. (n.d.). Noēsis: Plato on exact science, Unpublished.

Tait, W. (ed.) (1997). Early Analytic Philosophy: Frege, Russell, Wittgenstein. Essays in honor of Leonard Linsky, Chicago: Open Court. 